\title{
An Approach for Selecting Optimum Number of Base Stations and Optimizing Site Locations using Flower Pollination Algorithm
}

\author{
Ravneet Kaur \\ Yadavindra College of Engineering \\ Talwandi Saboo, Bathinda
}

\author{
Ashwani Kumar \\ Associate Prof., Yadavindra College of \\ Engineering \\ Talwandi Saboo, Bathinda
}

\begin{abstract}
Wireless communication has observed inordinate advancement since the beginning of this century. There is an expansion in wireless communication due to upsurgement in demands of customers for better services. To give fine quality to customers there is need to plan the network .This work considers how to optimally locate the BTS so that maximum coverage obtained at lesser infrastructure cost. This dissertation work is intended to present the investigations on swarm based optimization technique to locate the BTS in a network. In this work to locate the BTS, firstly calculate the SINR, capacity and network performance are calculated to find optimal no. of cell sites. Thereafter FPA is used to find the location of these sites. Furthermore FPA is compared with $\mathrm{ABC}$ to optimally locate the BTS.
\end{abstract}

\section{Keywords}

Flower Pollination Algorithm, Artificial Bee Colony Algorithm, Base Transceiver Station, Mobile Station, Cellular Mobile Communication.

\section{INTRODUCTION}

Wireless communication is one of the most swiftly enlarging industries over the universe. Whether it is cellular phones or the more traditional systems such as radio and television broad casting, wireless communication has become a fundamental part of infrastructure competiting other traditional infrastructures such as transportation, electric and water systems. Cellular wireless communication is facilitated by BTSs which have a suitable spatial distribution. Cell planning is a requisite and demanding part of cellular network design process [14]. The originally aim is the selection of BTS sites to provide maximum coverage while considering numerous baseline issues. For instance demand to cover a specific region, availability of BTS sites, available channel capacity at each BTS and the service quality at various potential Traffic Demand Areas (TDAs). There are several factors that are involved in design process of network that are coverage, traffic and system capacity. The location of cell can be determined on the basis of number of cells, the coverage performance, traffic distribution and the propagation environment. Various forms of inputs and cons from customers in terms of spectrum availability, network dimensions, frequency planning, network growth and finally $\mathrm{RF}$ environment plays a prominent role in coverage planning

When cellular concept was proposed, regular frequency reuse pattern is used for selection of BTS locations. The cost involved is setting up a network and the quality of service offered is directly proportional to the number of BTS installed, more BTS, more is the cost but better coverage at more infrastructure cost. During optimization the main parameters which are taken into consideration are power received, path loss and attenuation

\section{NETWORK PLANNING}

Network planning and design is an iterative process, surrounding topological design, network-synthesis, and network-realization, and directed at ensuring that a new telecommunications network or service assembles the requirements of the subscriber and operator. The factors that make BTS Planning a costly can be summed up as follows: firstly, if cell planning stage extends longer, then the overall development cost of the project goes up due to time dependency of the work. Secondly, the cost of setting up a new BTS includes the cost of hiring/buying land, RF cables, and shelter for equipment, antennas, power sources, and maintenance. Last but not least, the invaluable time factor that is involved for a perfect plan is also required.

\section{FLOWER POLLINATION ALGORITHM}

Pollination may be defined as the transmission of pollens from one flower to another flower in the same plant or another plant. This delivery of pollens can happen through pollinators such as birds, insects, bats and other animals. Flower pollen localization algorithm is a nature inspired algorithm which gets the idea from the peculiarity of flowering plants. The idea for localization was given by Xin-She Yang in 2012. Flower is used for reproduction of its own species through the process of pollination.

Pollination has two major types: abiotic and biotic. About $90 \%$ of flowering plants referred to biotic pollination. It means pollen is carried out by pollinators such as insects and animals. About $10 \%$ of pollination accepts abiotic form which does not need any pollinators. Wind as well as diffusion assists pollination of such flowering plants. For instance in grass there is abiotic pollination takes place. Pollination can be achieved by two processes.

\section{Self-Pollination \\ 2. Cross Pollination.}

\subsection{Self Pollination}

When the pollen from a flower pollinates the same flower or flowers of the identical plant, the process is called self pollination. It occurs when a flower contains both the male and the female gametes. 


\subsection{Cross Pollination}

Cross Pollination occurs when pollen grains are moved to a flower from another plant. The process of cross pollination happens with the help of abiotic or biotic agents such as insects, birds, snails, bats and other animals as pollinators. Abiotic pollination is a process where the pollination happens without involvement of external agents. Only about $10 \%$ of plants fall in this category. The process of pollination which requires external pollinators is known as Biotic Pollination to move the pollen from the anther to the stigma. Insects play most important role as the pollinators. Insect Pollination occurs in plants with coloured petals and strong odour which attract Honey bees, moths, beetles, wasps, ants and butterflies. The insects are attracted to flowers due to availability of nectar, edible pollen and when insect sits on the flower, the pollen grains stick to the body. When the insect visits another flower, the pollen is transferred to stigma facilitating pollination. The pollination is also facilitated by vertebrates like birds and bats. Flowers pollinated by bats mostly have white coloured petals and strong odour. The birds usually pollinate flowers with red petals and without odour.

\subsection{Rules}

Based on the above characteristics of flower pollination, XinShe Yang developed the Flower pollination algorithm (FPA). For simplicity some rules are there:

1. Biotic and cross-pollination can be considered as a process of global pollination process, and pollencarrying pollinators move in a way which obeys L'evy flights (Rule 1).

2. For local pollination, abiotic and self-pollination are used (Rule 2).

3. Pollinators such as insects can develop flower constancy, which is equivalent to a reproduction probability that is proportional to the similarity of two flowers involved (Rule 3).

4. The interaction or switching of local pollination and global pollination can be controlled by a switch probability $p[0,1]$, with a slight bias towards local pollination (Rule 4)

\section{ARTIFICIAL BEE COLONY ALGORITHM}

The artificial bee colony algorithm is a type of an optimization algorithm which is used network planning based on the intelligent foraging expedition behaviour of honey bee swarm. This method was suggested by Karaboga in the year 2005.In the $\mathrm{ABC}$ algorithm, there is a colony which considers three groups of bees: employed bees, onlooker bees and scout bees. It is supposed that there is only one artificial employed bee for each diet source. In other words, the number of employed bees which are present in the colony is alike the number of diet sources around the hive. Employed bees go to search food source and come back to hive and dance on the particular region. The employed bee whose food source has been relinquished becomes a scout bee. Furthermore, departs to scrutinize for discovery of a new food source. As far as Onlookers are concerned they only watch the dances of employed bees and pick out food sources depending on dances.

\section{OBJECTIVES}

The objective of thesis is to optimally locate BTS so that minimum number of BTS can be installed at lesser infrastructure cost with minimum interference. The main problem is to optimize location of BTS with respect to each MS using algorithms. The parameters are: power received path loss and attenuation. It means in this work the main aim is to reduce path loss, attenuation and good power received so that there is maximum coverage

The fitness of solution is decided according to Hata Model Equation [8]

$$
\begin{aligned}
& \mathrm{L}_{\mathrm{p}}=69.55+26.16_{\log }(\mathrm{f})-13.82 \log \left(\mathrm{H}_{\mathrm{bts}}\right)-\mathrm{a}_{\mathrm{m}}\left(\mathrm{h}_{\mathrm{m}}\right)+\{44.9- \\
& 6.55 \log (\underset{\mathrm{bts}}{\mathrm{h}})\} \log (\mathrm{d})+\mathrm{Qo} \\
& \mathrm{A}=42.6+20 \log \mathrm{f}+26 \log \mathrm{d}
\end{aligned}
$$

\section{OPTIMIZED ALGORITHM}

In this thesis the main aim is to provide proper network coverage by algorithms. Firstly two inputs are taken initially in the form of maximum number of base stations as well as number of mobile stations to be served. Thereafter; capacity, SINR and performance will be calculated. After proceeding this according to SINR ranking is done so that the BSs which are not in use will be eliminated to serve the mobile stations with proper coverage. Moreover screening of base stations is done to avoid the interference of traffic. The whole scenario is optimized in MATLAB.Once the cell site locations are optimized then apply $\mathrm{ABC}$ and FPA on the resultant Base stations .First of all calculation of power received, path loss and attenuation is carried out with ABC and FPA algorithm individually. In this subsequent area the fitness function is calculated. To achieve the maximum coverage optimization is done. Furthermore, relevant comparisons of two algorithms i.e. artificial bee colony optimization and flower pollination are taken with respect to path loss, power received and attenuation. In addition to this, requisite graphs are drawn on the basis of comparison between $\mathrm{ABC}$ and FPA so as to understand the whole process. The flow diagram of fitness algorithm is as follows: 

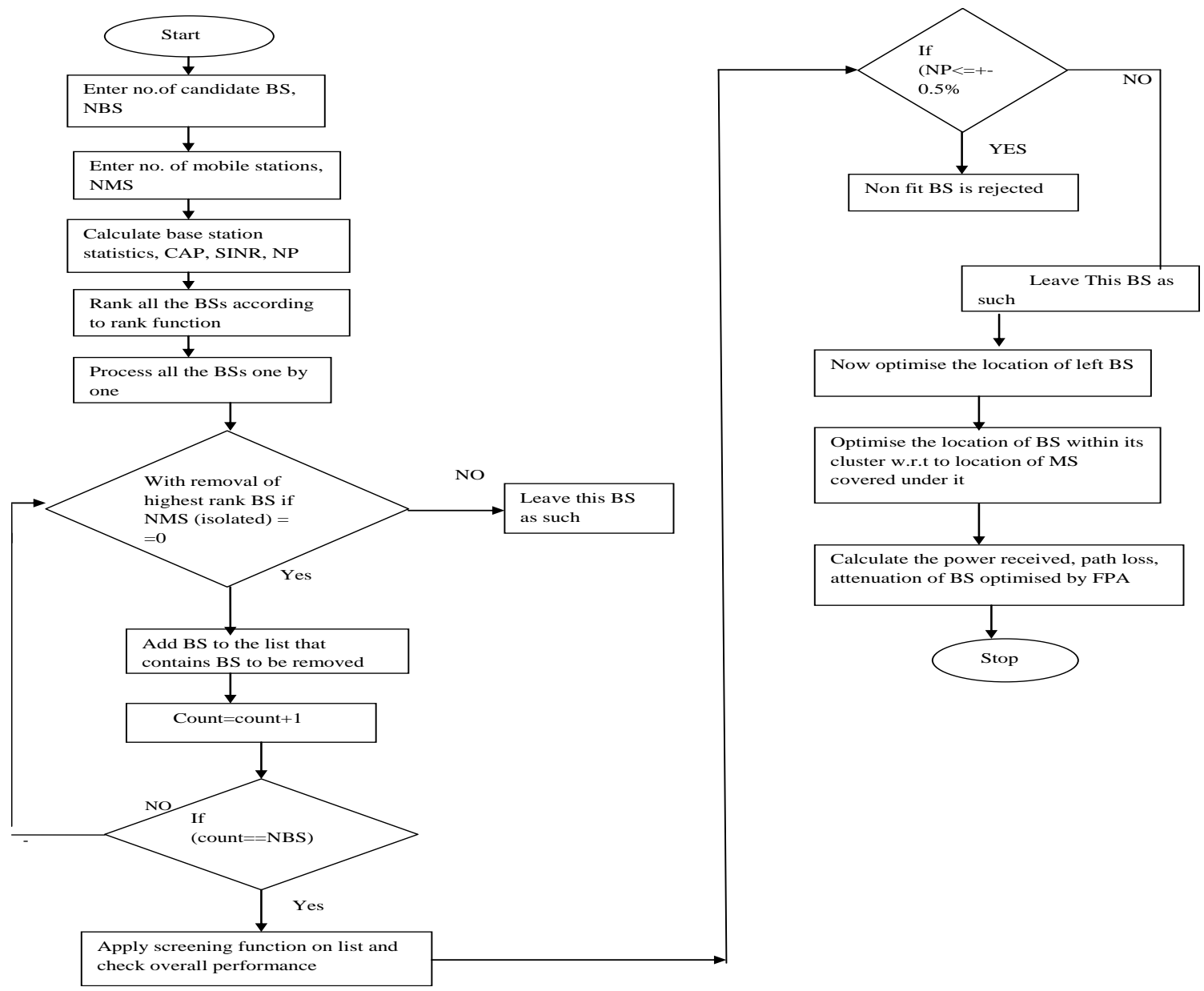

Fig: flow chart of optimized network planning of BTS

\section{SIMULATION RESULTS}

By analysing the both algorithms ABC and FPA following results are observed. From the collation it is noted that flower pollination gives better results as compare to artificial bee colony algorithm. Various figures are created in the MATLAB environment. Firstly number of base stations and mobile station locations are generated randomly before optimization. The figure is shown as below:

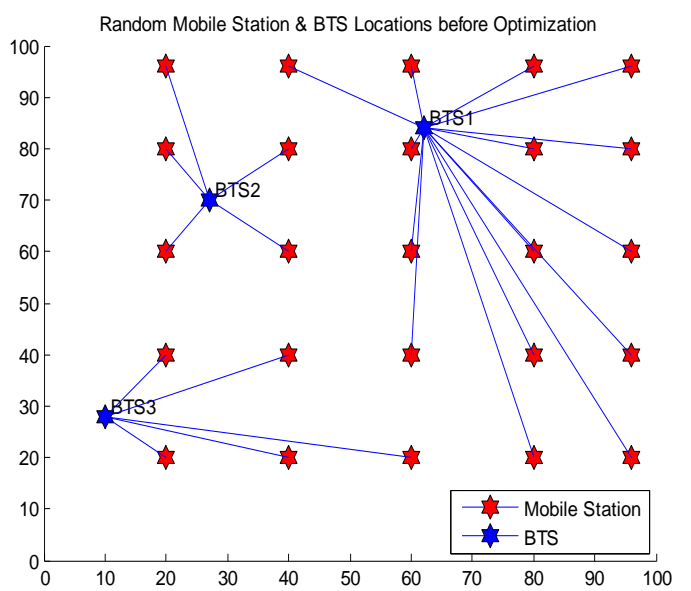

Figure7.1 Random locations of base station and mobile station before optimization
The next phase is to apply the $\mathrm{ABC}$ algorithm on the left cell site location. After the whole processing of $\mathrm{ABC}$ algorithm, the optimized location of BTS are calculated so that maximum coverage is observed.

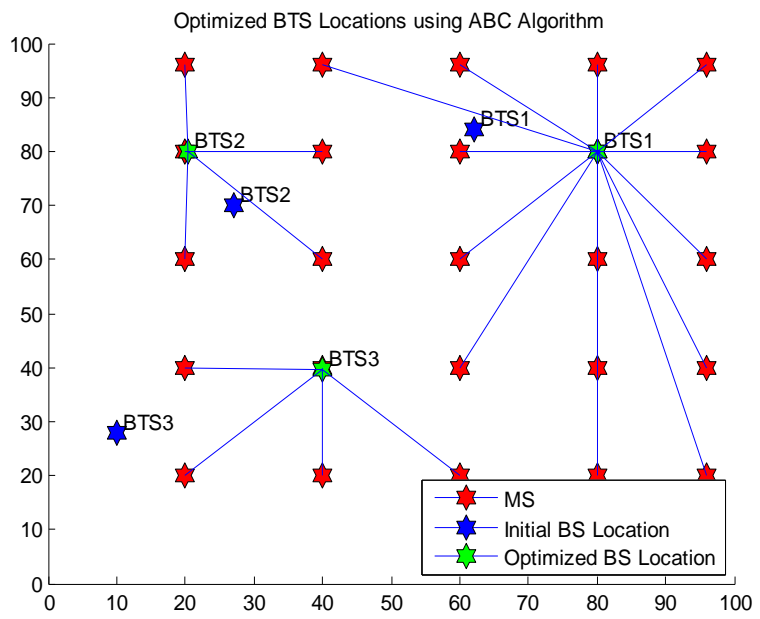

Figure 7.2 Base station locations after optimization using $\mathrm{ABC}$ algorithm

The aim of this thesis is to calculate power received, path loss and attenuation. The bar graph below shows the respective parameters. 


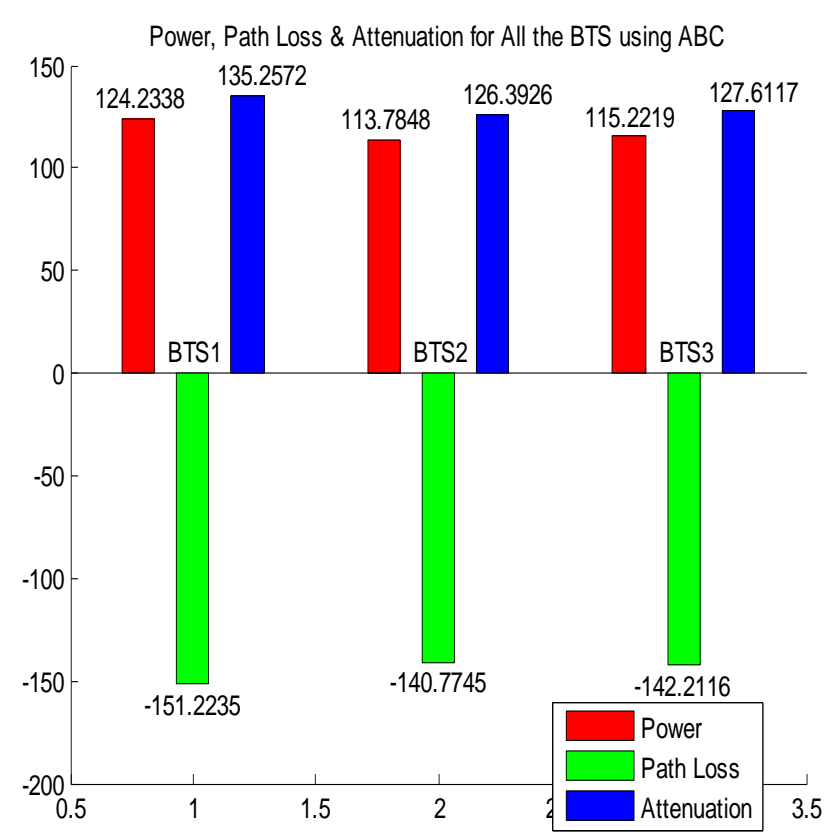

Figure 7.3 Graphical representation of power received, path loss and attenuation using artificial bee colony algorithm

After this, optimization is done with the help of Flower pollination algorithm. In this firstly, random locations are concluded as like ABC.Thereafter during optimization, after optimization processes are taken into consideration. The graphical representation is as follows

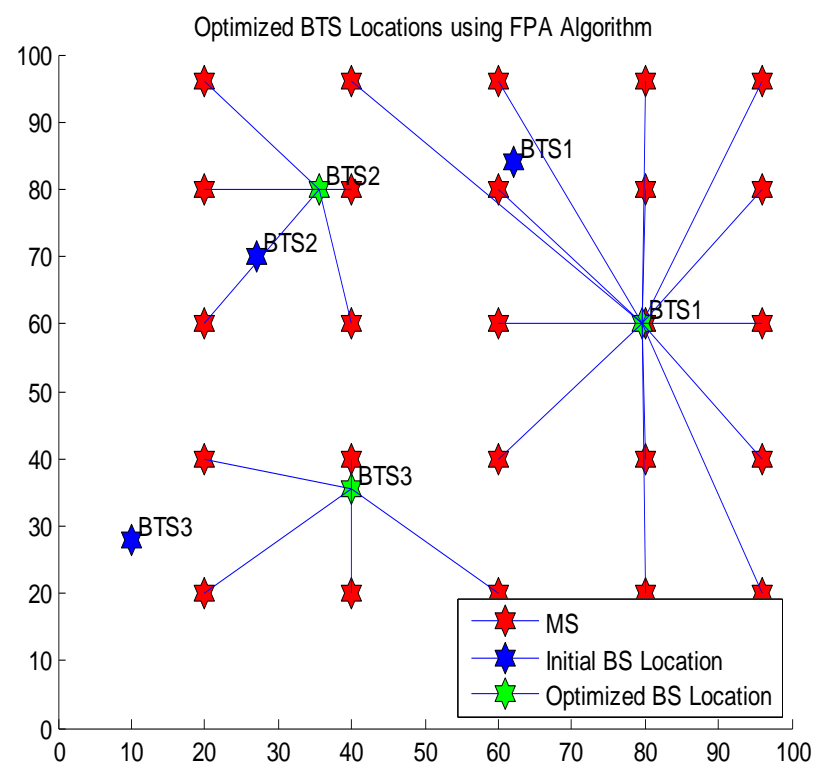

Figure 7.4 Optimized locations of BTS using FPA

The path loss, Power received and attenuation shows better results as compare to $\mathrm{ABC}$. The outline of the bar graph is given under

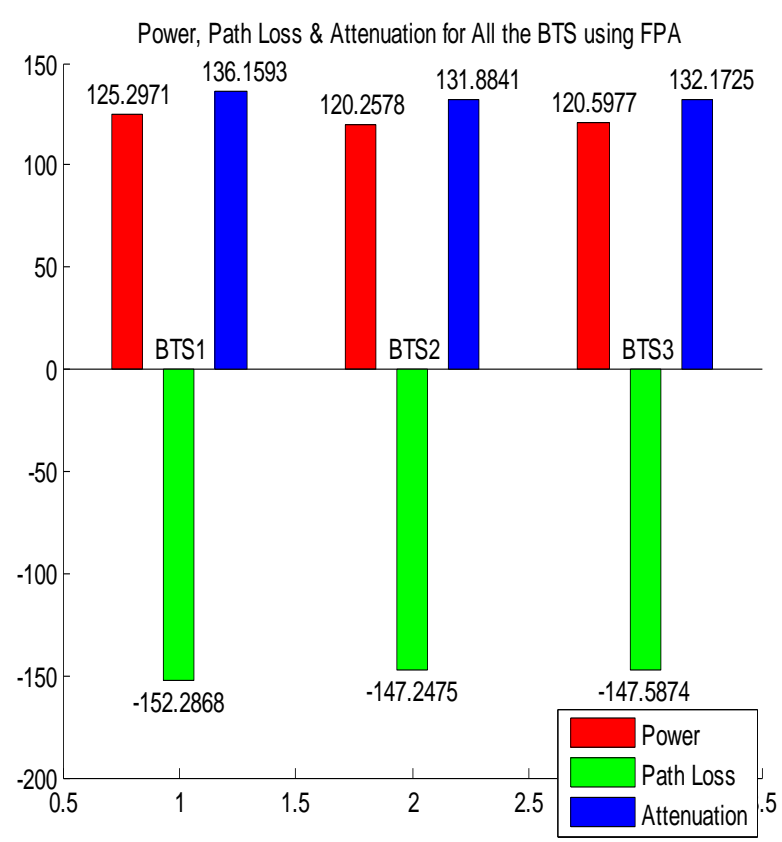

Figure 7.5 Bar graph showing factors using FPA algorithms

The combined average comparison of power received, path loss and attenuation can be shown graphically. In this phase the results of $\mathrm{ABC}$ and FPA. The bar graph representation of the average comparisons are given below which clearly represent the fundamental collation of these parameters.

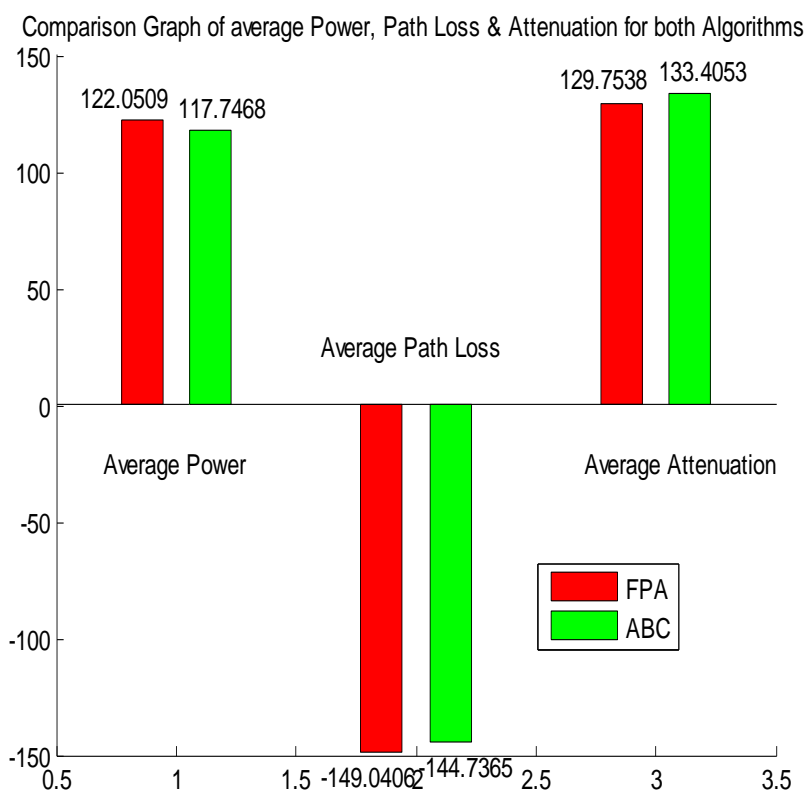

Figure 7.6: Average comparison of power, path loss and attenuation for $\mathrm{ABC}$ and FPA 


\section{TABULAR REPRESENTATIONS OF RESULT \\ Table 1: Tabular representation of parameters}

\begin{tabular}{|l|l|l|}
\hline Parameters & Using ABC & Using FPA \\
\hline Power received & 117.7468 & 122.0509 \\
\hline Path Loss & -144.736 & -149.406 \\
\hline Attenuation & 133.4053 & 129.7538 \\
\hline
\end{tabular}

\section{CONCLUSION AND FUTURE SCOPE}

Research is an iterative process very similar to artificial bee colony algorithm and flower pollination algorithm where researchers keep on proposing and implementing new ideas based on their previous successes and the successes observed by other researchers in the area. Various research observations

are presented at the end of previous chapter as conclusions but limited to scope of this thesis only. This chapter aims to conclude the thesis as a whole, and to aggregate all the offshoots found throughout the work.

It is a novel approach which is intended to get the proper network by various techniques like artificial bee colony, flower pollination algorithm .To attain good network design optimization algorithms are mandatory so as maximum coverage is obtained. This work represents the optimally location of cell sites as well as calculating the base stations according to signal to noise ratio, capacity. Moreover this dissertation work also successfully locate the base stations according to differ algorithms for instance artificial bee colony and flower pollination. After implementing the algorithms requisite comparisons are done between two algorithms to check the proper location of base transceiver station. The factors considered are path loss, power received and attenuation. In addition to it, this dissertation work successfully compares the two algorithms and provides required coverage to the users. Furthermore, in this work the performance of flower pollination is better achieved than artificial bee colony algorithm. The proposed work has ability to achieve optimal solution of coverage problem with minimum number of base stations in cellular networks. This approach cultivates an innovative idea on employing the flower pollination algorithm with enhanced coverage. The results show that the flower pollination approach is effective and robust for efficient coverage problem of base transceiver station location and is considered to give almost the optimal solution in wireless communication network.

In future, focus can be given to achieve $100 \%$ coverage with minimum number of BTS. The study of the $100 \%$ coverage using various optimal search techniques also presents several interesting challenges. These problems are over complex, therefore their solution can be considered as a very good indicator for the potential of the nature inspired algorithms including artificial bee colony and flower pollination algorithms. This research can be further optimized using other algorithms like ant colony, spider monkey algorithm to achieve maximum power received, minimum path loss and attenuation.

\section{ACKNOWLEDGEMENT}

I express my gratitude to all people who help me in my work especially to my honourable guide at $\mathrm{YCoE}$ Talwandi Saboo, Sh. Ashwani Kumar (Associate Professor) who helped me in my work.

\section{REFERENCES}

[1] Abdul Ghani Abro, Junita Mohamad-Saleh "Intelligent Scout-Bee Based Artificial Bee Colony Optimization Algorithm" International Conference on Control System, Computing and Engineering, 23 - 25 Nov. 2012, Penang, Malaysia IEEE 2012

[2] Aida Al-Samawi, AduwatiSali, NorKamariahNoordin, Mohamed Othman , and Fazirulhisyam Hashi, "Base Station Location Optimization in LTE using GA",Dept. of Computer \& Communication Systems Engineering Faculty of Engineering, UPM, 43400, Selangor, Malaysia , Department of Communication Technology and Network Faculty of Computer Science and Information Technology, UPM, Selangor, Malaysia 2013 IEEE.

[3] Amar Pratap Singh.J, Karnan.M, "Using a Novel Intelligent Location Management Strategy in Cellular Networks, Research Scholar, Computer Science and Engineering Anna University Coimbatore, 2010 International Conference on Signal Acquisition and Processing

[4] Ankita Awasthi, Neha Arora, "An Approach to BTS Localization using Optimization Techniques" Dept. of Telecom Engineering, AITEM ,Amity University, Noida, India. International Journal of Engineering Research \& Technology (IJERT) ISSN: 2278-0181Vol. 3 Issue 4, April - 2014.

[5] David Abusch-Magder "Novel Algorithms for Reducing Cell Sites During a Technology Upgrade and Network Overlay" Wireless Technology Lab, Bell Labs, Lucent Technologies 600 Mountain Ave, Murray Hill, NJ 07974 IEEE 2005

[6] Kamalam Balasubramani, Karnan Marcus "A Study on Flower Pollination Algorithm and Its Applications" Associate Professor, Dept. of ISE Atria Institute of Technology Bangalore, India, International Journal of Application or Innovation in Engineering \& Management (IJAIEM), Volume 3, Issue 11, November 2014

[7] Liza K. Pujji - Kevin W. Sowerby · Michael J. Neve, "Development of hybrid algorithm for efficient optimization of base station placement for indoor wireless communication system" Department of Electrical and Computer Engineering, The University of Auckland,Auckland, New Zealand, Springer 2013

[8] M. Hata. Empirical formula for propagation loss in land mobile radio services. Vehicular Technology, IEEE Transactions on, 29(3):317-325, 1980

[9] Rambally, R.S, Maharajh, A, "Cell Planning Using GA and Tabu Search" ; Univ. of Trinidad \& Tobago 2009 IEEE

[10] Satvir Singh, Kulvinder Kaur, "Base Station Localization using Artificial bee colony Algorithm" SBS State Technical Campus Ferozepur, Punjab, INDIA 
International Journal of Computer Applications (0975 8887) Volume 64 - No. 9, February 2013.

[11] Williamjeet Singh and Jyotsna Sengupta, "Optimal Cell Site Planning in Varying Geographic Regions for Cellular Networks", International Journal of Computer Applications in Engineering, Technology and Sciences (IJ-CA-ETS), Volume 3: Issue 1, Oct 2010 - March 2011.

[12] Williamjeet Singh and Jyotsna Sengupta, "Performance Estimation of a Cell Site in Cellular Networks Using WiMAX Technology", International Journal of Information Sciences and Application, Volume 2: Number 3, November, 2010.

[13] Williamjeet Singh and Jyotsna Sengupta, "Performance Estimation of a Cell Site in Cellular Networks Using WiMAX Technology", International Journal of Theoretical and Applied Information Technology, Volume 28: Number 1, June, 2011.

[14] Williamjeet Singh, Jyotsna Sengupta “An Optimized Approach for Selecting an Optimal No. Of Cells Site Location In Cellular Network" PhD Scholar UCoE
Punjabi University Patiala,Punjab,International Journal of Computer Applications, Volume 40-no.8,Feburary 2012

[15] Xin-She Yanga, Mehmet Karamanoglua, Xingshi Heb "Multi-objective Flower Algorithm for Optimization" a School of Science and Technology, Middlesex University, London NW4 4BT, UK school of Science, Xi'an Polytechnic University, Xi'an, P. R. China ICCS 2013

[16] Y. Xin-She, "Nature-inspired Metaheuristic Algorithms," Luniver Press, 2008.

[17] Y. Xin-She, Engineering Optimization: An Introduction with Metaheuristic Application, Wiley, 2010.

[18] Zhang Dongli ,Guan Xinping ,Tang Yinggan,Tang Yong,"An Artificial Bee Colony Optimization Algorithm based on Multi-exchange Neighbourhood", Institute of Electrical Engineering Yanshan University Qinhuangdao, China, Fourth International Conference on Computational and Information Sciences IEEE 2012

[19] T. S. Rappaport, "Wireless Communications", 2nd Edition, Prentice Hall, Chapter 4, 2002. 